\title{
Three-dimensional ophthalmic artery model reconstruction and assessment captured by computed tomographic angiography in non-arteritic anterior ischemic optic neuropathy patients
}

\author{
Jia-Lin Wang^, Dong-Hui Wu^, Hong-Yang $\mathrm{Li}^{\#} \wedge$, Yan-Ling Wang ${ }^{\#} \wedge$ \\ Department of Ophthalmology, Beijing Friendship Hospital, Capital Medical University, Beijing, China \\ Contributions: (I) Conception and design: JL Wang, HY Li; (II) Administrative support: YL Wang; (III) Provision of study materials or patients: YL \\ Wang, HY Li; (IV) Collection and assembly of data: JL Wang, DH Wu; (V) Data analysis and interpretation: JL Wang; (VI) Manuscript writing: All \\ authors; (VII) Final approval of manuscript: All authors. \\ "These authors contributed equally to this work. \\ Correspondence to: Yan-Ling Wang; Hong-Yang Li. Department of Ophthalmology, Beijing Friendship Hospital, Capital Medical University, Beijing \\ 100050, China. Email: wangyanl@ccmu.edu.cn; faraway_sweet@163.com.
}

\begin{abstract}
Background: The ophthalmic artery (OA) was first reconstructed using computer software. The structural differences of ophthalmic arteries in non-arteritic anterior ischemic optic neuropathy (NAION) and normal eyes, in addition to hemodynamic alterations, were assessed.

Methods: Thirty-one NAION eyes, 19 uninvolved eyes with NAION, and a control group of 26 healthy eyes were retrospectively included. Computed tomographic angiography data were recorded, and corresponding three-dimensional OA models were constructed. Initial OA and internal carotid artery (ICA) diameters and the angle between them were analyzed. Three different OA models were used to evaluate hemodynamic performance. The statistical relationships between the initial diameters of the OA and ICA and the angle between the OA and ICA were described.

Results: OA diameters in NAION eyes were significantly smaller than those in both uninvolved and healthy eyes $(\mathrm{P}<0.05)$. There was no significant difference between uninvolved and healthy eyes $(\mathrm{P}=0.31)$. The initial ICA diameter and the angle between the OA and ICA did not significantly differ among the three groups. In the three models, the blood flow velocity in the initial ophthalmic arteries of uninvolved eyes was higher than that in the NAION eyes. The mass flows of the right and left ophthalmic arteries, accounting for the ipsilateral ICA in the control model, were $0.57 \%$. However, these values in uninvolved and NAION eyes were $1.36 \%$ and $0.25 \%$, respectively.

Conclusions: NAION is associated with a smaller initial OA diameter, which may be related to hypoperfusion. To our knowledge, this is the first pilot study to analyze hemodynamic alterations using OA models.
\end{abstract}

Keywords: Non-arteritic anterior ischemic optic neuropathy (NAION); ophthalmic artery (OA); threedimensional reconstruction; hemodynamic performance

Submitted Oct 13, 2020. Accepted for publication Dec 24, 2020.

doi: 10.21037/atm-20-6883

View this article at: http://dx.doi.org/10.21037/atm-20-6883

^ ORCID: Jia-Lin Wang, 0000-0001-7877-1620; Dong-Hui Wu, 0000-0003-3576-0221; Hong-Yang Li, 0000-0001-6786-2105; Yan-Ling Wang, 0000-0002-7191-6660. 


\section{Introduction}

Non-arteritic anterior ischemic optic neuropathy (NAION) is a disease characterized by acute, painless optic disc swelling with accompanying visual loss or visual field defects (1). It is the most common type of acute optic neuropathy and induces severe visual impairment in individuals over the age of 50 years (2). Although various etiologies have been reported to cause NAION, the exact pathogenic mechanism remains unclear. NAION appears to be a multifactorial disease (3). It is most frequently caused by transient non-perfusion (4) or hypoperfusion of the optic nerve head at the level of the short posterior ciliary arteries. This alteration in perfusion leads to focal compartment syndrome, which induces further ischemia. The exact cause of the transient disturbance, however, remains unclear. Several hypotheses have been proposed, including vasospasm, autoregulation failure, venous occlusion, and thrombosis. In addition, a small crowded disc is referred to as a "structural disc at risk" for NAION.

To date, no study has investigated the structural differences and blood flow alterations of the ophthalmic artery (OA) between NAION and normal eyes. Color Doppler imaging (CDI) is widely used to investigate retrobulbar vascular parameters, including blood velocity, pulsatility index, and resistive index, in both healthy and diseased eyes (5). However, CDI has inherent limitations; it is not a reliable diagnostic method for visualizing and calculating the diameter of the OA, because it is difficult to capture the origin of the $\mathrm{OA}$ and visualize the morphological changes in the distal part of the artery. In addition, the accuracy of CDI is influenced by the skill of the examiner (6). Digital subtraction angiography is the gold-standard modality for visualizing relatively small vessels, but it is an invasive diagnostic method with significant stroke-related morbidity because of embolic phenomena, especially in older patients with atherosclerotic vessels (7). Computed tomography angiography (CTA) has become the first-line method, with an increasing number of neurovascular imaging applications. The increase in the number of image slices per study has enabled the clear depiction of complex vascular trees and their nearby structures (8).

Computer-based simulation has been used to generate three-dimensional (3D) models and clarify the hemodynamics in patients with brain aneurysms, coronary artery aneurysms, and abdominal aortic aneurysms, among other conditions (9-11). However, it has not been used in ocular diseases. It could be important for ophthalmologists to further explore the pathogenic mechanisms of NAION to overcome the abovementioned limitations experienced in the investigation of morphological changes and flow alterations in the OA in patients with NAION. Thereafter, we determined the quantitative data of structural differences by comparing normal and NAION eyes, and we evaluated the blood flow alterations of the OA. This is the first preliminary study to analyze the structural and hemodynamic alterations of OAs using computer-based simulation models.

We present the following article in accordance with the STROBE reporting checklist (available at http://dx.doi. org/10.21037/atm-20-6883).

\section{Methods}

\section{Participants}

The study was conducted in accordance with the Declaration of Helsinki (as revised in 2013). The study was approved by the ethics board of Beijing Friendship Hospital affiliated with Capital Medical University Ethics Committee (No. 2020-P2-008-01) and informed consent was taken from all individual participants.

Patients with both healthy and NAION eyes who visited Beijing Friendship Hospital were retrospectively identified. All patients who were diagnosed with NAION between January 2017 and January 2019 were considered for our study. Patients in our study included bilateral NAION patients. Patients whose initial OA could not be visualized by CTA were excluded from our study. The included patients were stratified into three groups: healthy, uninvolved, and NAION. Healthy eyes without NAION, fundus disease, or history of laser therapy or eye surgery were assigned to the healthy group. The uninvolved group included 19 contralateral eyes without a diagnosis of NAION. The NAION group included 31 eyes based on the following inclusion criteria (12): (I) a history of sudden visual loss, typically experienced in the morning, with an absence of other ocular and neurologic diseases that may better explain the patient's visual symptoms; (II) optic disc edema at the onset of vision loss that was documented by at least two ophthalmologists; (III) optic disc edema that spontaneously resolved within 2-3 months; (IV) visual field defects associated with the optic disc in the affected eye; and (V) no neurologic or ocular disorder that could be the underlying cause of the optic disc edema and visual 


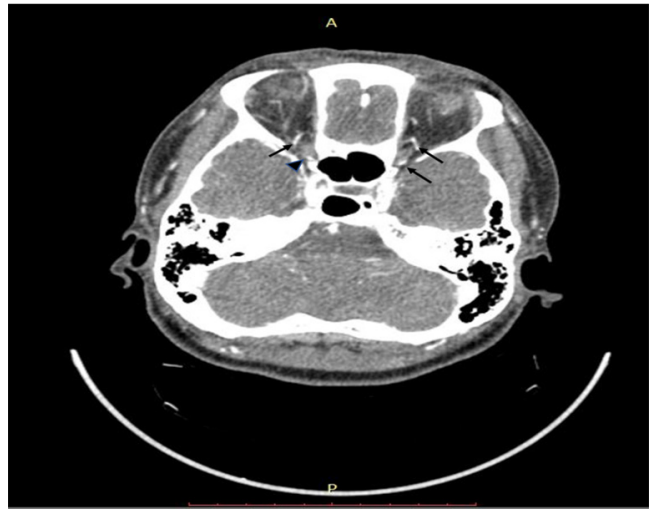

Figure 1 Representative CTA image of an OA. Axial section image at the origin of the artery (black triangle). The black arrow shows the OA position. OA, ophthalmic artery; CTA, computed tomography angiography.

field defects. The exclusion criteria were: (I) previous corticosteroid therapy or any other treatment for NAION; (II) arteritic anterior ischemic optic neuropathy (13); and (III) cranial CT, carotid artery Doppler, or cranial magnetic resonance imaging showing a disease that would result in insufficiency in OA blood supply, such as brain tumor compression or carotid artery stenosis. In total, 76 eyes from patients who underwent head-and-neck CTA evaluation were enrolled. The healthy group consisted of 26 eyes from healthy age-matched subjects with no evidence of ophthalmic pathology, who underwent head-and-neck CTA because of other symptoms and had normal findings.

\section{Head-and-neck CTA}

The CTA scans were performed by a 64-row multidetector CT scanner (Light Speed VCT, GE Healthcare; Chicago, IL). Scans extended from the aortic arch to the skull base. CT parameters were as follows: pitch, 0.984; slice thickness, $0.9 \mathrm{~mm}$; reconstruction interval, $0.7 \mathrm{~mm}$. A representative OA CTA image is shown in Figure 1.

\section{Ophthalmic artery model}

To simulate the actual hemodynamic conditions of the patients' OAs, arterial models were reconstructed based on CTA findings obtained from Beijing Friendship Hospital. These images were used to reconstruct models using Mimics software (v15.0, Materialise NV; Leuven, Belgium). Simple smooth processing was applied using Geomagic
Studio v12.0 (3D Systems, Rock Hill, SC). Typical OA models of healthy controls and patients with NAION (i.e., those with one eye in the NAION group, and the other eye in the uninvolved group) are shown in Figure 2.

Mimics software was used to measure the diameter of the initial OA, the diameter of the internal carotid artery (ICA), and the angle between the OA and the ICA (Figure 3).

\section{Mesh generation}

To generate the patient models, we used the original models for the healthy controls and NAION patients. To calculate the mass flow, meshes were generated using ANSYS ICEM CFD (ANSYS, Canonsburg, PA). The surfaces of the models were meshed using a mixture of tetrahedral and triangular prism volume meshes. Altogether, there were 266,871 and 240,381 nodes for the right and left sides for the healthy control patient model, respectively. There were 198,443 and 179,154 nodes for the uninvolved and NAION sides in the model generated using CTA data from NAION patients, respectively.

\section{Boundary conditions}

The mean blood velocity for the typical group models at the inlet was $0.34 \mathrm{~m} / \mathrm{s}$ (10). For simplicity, pressure boundary conditions with $\mathrm{P}=0 \mathrm{~Pa}$ were applied at the outlets. Identical boundary conditions and model parameters were used in all models.

\section{Assumptions and governing equations}

A finite volume method for steady flow was used in the simulation using ANSYS ICEM CFD (ANSYS) (11). Discretization of the pressure and momentum at each control volume was in a second-order scheme. The iterative process of computation was terminated when the residual of continuity and velocity were both less than the convergence criterion, $1.0 \times 10^{-5}$. A pressure-based solver was used with pressure modification. The blood was assumed an incompressible, homogenous, and Newtonian fluid $(14,15)$. The numerical simulation was based on the following $3 \mathrm{D}$ incompressible Navier-Stokes equation and continuity condition:

$$
\begin{aligned}
& \rho(\vec{u} \cdot \nabla) \vec{u}+\nabla p-\mu \Delta \vec{u}=0 \\
& \nabla \cdot \vec{u}=0
\end{aligned}
$$



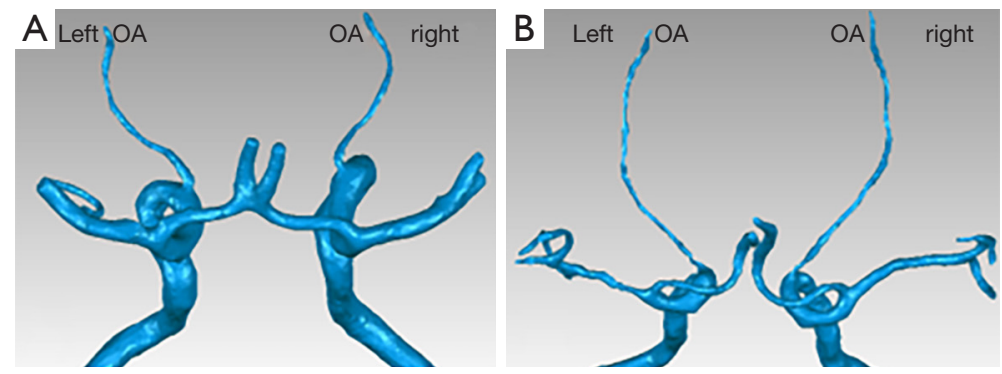

Figure 2 Typical OA models reconstructed from CTA data. (A) Healthy controls; (B) NAION group (right, uninvolved eye; left, NAION eye). OA, ophthalmic artery; CTA, computed tomography angiography; NAION, non-arteritic anterior ischemic optic neuropathy.

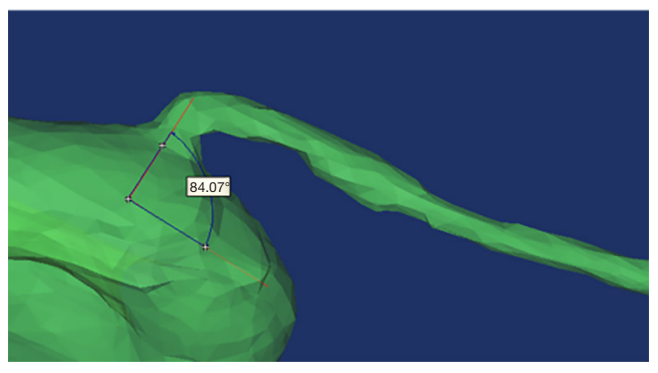

Figure 3 Angle between the OA and ICA of a healthy control patient. OA, ophthalmic artery; ICA, internal carotid artery.

\section{Statistical analysis}

Data are presented as means \pm standard deviations. Statistical analysis was performed using SPSS for Windows, version 24.0 (IBM, Armonk, NY). Statistical evaluations were performed using a one-way analysis of variance. Categorical data were analyzed using Fisher's exact test and chi-squared tests. A P value less than 0.05 was considered statistically significant.

\section{Results}

This study included 26 healthy eyes, 19 uninvolved eyes, and 31 NAION eyes with initial OAs that could be visualized on CTA. Age, sex, diabetes, and hypertension showed no statistically significant differences between groups (Table 1).

\section{Quantitative analysis of the $\mathrm{OA}$ models}

Table 2 shows the results of quantitative analysis of the three OA models. The diameter of the OA in NAION eyes was significantly smaller than that of uninvolved eyes in patients with NAION and healthy controls $(\mathrm{P}<0.05)$, and there was no significant difference between uninvolved eyes and healthy eyes $(\mathrm{P}=0.31)$. The diameter of the initial ICA and the angle between the OA and ICA were not different among the three groups.

\section{Flow velocity (flow pattern)}

Figure 4 shows the $3 \mathrm{D}$ velocity streamlines in the typical models, with magnitudes of flow velocity indicated by color.

Although there was a difference in ICA flow between the left and right sides because of differences in ICA inlet diameters, there was no obvious difference in blood flow velocity in the OA between the right and left sides of healthy control subjects. However, blood flow velocity in the original OA of the uninvolved eye was higher than that of the NAION eye.

\section{Mass flow}

To explore blood flow in the OA on the NAION side with more accuracy, we analyzed the proportion of OA blood flow in each model. The mass flows of the right and left sides of the $\mathrm{OA}$ in healthy controls were $0.57 \%$ of the ipsilateral ICA mass flow. However, the mass flows of the uninvolved and NAION sides of the OA were $1.36 \%$ and $0.25 \%$ of the ipsilateral ICA mass flow, respectively. For patients with NAION, the mass flow of the NAIONside OA, which accounted for the ipsilateral ICA mass flow, was much lower than that of the uninvolved side. In addition, the mass flow of the OA proportion of the NAION side was lower than that of healthy controls. Figure 5 shows the inlet and outlet mass flows in the different patient models. 
Table 1 Demographic characteristics of all patients

\begin{tabular}{|c|c|c|c|c|}
\hline Variables & Healthy eyes $(n=26)$ & Uninvolved eyes $(n=19)$ & NAION eyes $(n=31)$ & $P$ value \\
\hline Sex, male/female & $11 / 15$ & $9 / 10$ & $13 / 18$ & 0.922 \\
\hline History of diabetes, n (\%) & $14(53.85)$ & $12(63.16)$ & $17(54.84)$ & 0.798 \\
\hline History of hypertension, n (\%) & $12(46.15)$ & $9(47.37)$ & $14(45.16)$ & 0.988 \\
\hline
\end{tabular}

Statistical significance tested by analysis of variance for normal distributions and Kruskal-Wallis tests for non-normal distributions; all comparisons were corrected with a post hoc test. Categorical data were analyzed using Fisher's exact test or chi-squared tests. No statistically significant differences were found among the groups. NAION, non-arteritic anterior ischemic optic neuropathy.

Table 2 Quantitative analysis of ophthalmic artery models

\begin{tabular}{lcccc}
\hline Variables & Healthy eyes $(n=26)$ & Uninvolved eyes $(n=19)$ & NAION eyes $(n=31)$ & $P$ value \\
\hline Initial diameter of OA $(\mathrm{mm})$ & $1.39 \pm 0.15$ & $1.34 \pm 0.17$ & $1.17 \pm 0.20$ & $<0.001^{*, * \star}$ \\
Angle between ICA and OA $\left(^{\circ}\right)$ & $71.41 \pm 16.93$ & $65.49 \pm 14.74$ & $70.15 \pm 14.52$ & 0.424 \\
Initial diameter of ICA $(\mathrm{mm})$ & $7.44 \pm 0.95$ & $7.98 \pm 1.60$ & $7.99 \pm 1.43$ & 0.233 \\
\hline
\end{tabular}

*, significant difference between mean values of uninvolved eyes and NAION eyes; ${ }^{*}$, significant difference between mean values of healthy eyes and NAION eyes. OA, ophthalmic artery; ICA, internal carotid artery; NAION, non-arteritic anterior ischemic optic neuropathy.
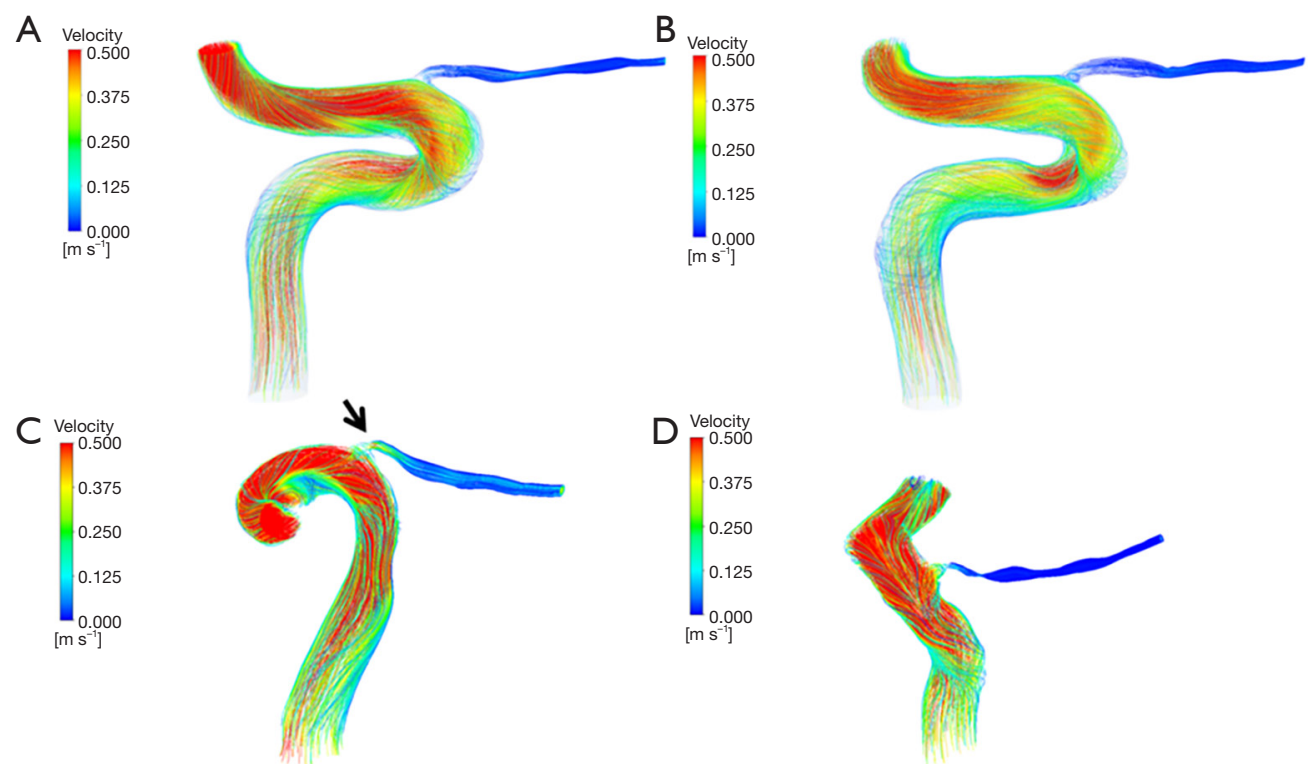

Figure 4 The streamlines are colored according to the magnitude of velocity in two typical patients. (A) Right side of a healthy control subject, (B) left side of a healthy control subject, (C) uninvolved side of a patient with NAION, and (D) NAION side of a patient with NAION. The flow velocity of the uninvolved side was higher (arrow) than the NAION side. NAION, non-arteritic anterior ischemic optic neuropathy. 

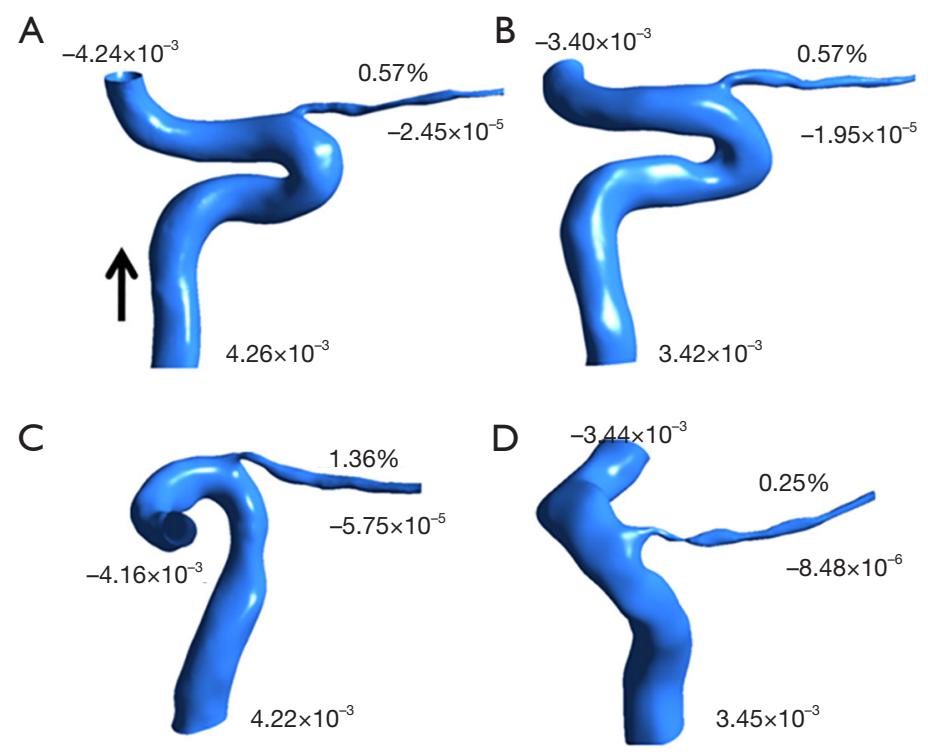

Figure 5 Mass flow $(\mathrm{kg} / \mathrm{s})$ of a healthy control model and a NAION patient model. (A) Right side of a healthy control subject, (B) left side of a healthy control subject; (C) uninvolved side of a patient with NAION, and (D) NAION side of a patient with NAION. Inlet (+), outlet (-). Blood flow direction (black arrow). NAION, non-arteritic anterior ischemic optic neuropathy.

\section{Discussion}

In this study, we generated a 3D OA model using CTA data to simulate the in vivo morphology and anatomy of OA. We analyzed and compared the OA diameter and the angle between the OA and ICA between healthy controls and patients with NAION. To explore the exact mechanism of NAION, we investigated blood flow alterations of computer-generated models of healthy controls and patients with NAION.

The anatomy of the OA has been described in many studies $(16,17)$, but these descriptions are inconsistent. We measured the diameter of the initial OA, which arises from the ICA. In our study, the diameter of the initial OA was $1.39 \pm 0.15 \mathrm{~mm}$ in healthy controls, $1.34 \pm 0.17 \mathrm{~mm}$ in uninvolved eyes of patients with NAION, and $1.17 \pm 0.20 \mathrm{~mm}$ in the NAION eyes of patients with NAION. For the healthy control subjects examined in our study, the values of initial OA diameter differed slightly from those reported by Jiménez-Castellanos et al. (18), who measured the diameter after perfusion, fixation, and dehydration, which induced major differences in the elasticity and diameter of the vessels. In contrast, we measured the in vivo inner diameter of the vessel using a computer-generated 3D method. The difference between our results and those reported by Zhang et al. (19) may be due to different measurement locations of the OA in healthy people.

To the best of our knowledge, the OA is narrow, tortuous, and difficult to measure, and previous NAION studies have focused on the analysis of the ICA and posterior cerebral artery. This is the first study in which the diameter of the OA was measured and analyzed in patients with NAION. In our study, the diameter of the OA in NAION eyes was significantly smaller than that of the uninvolved eyes in patients with NAION and healthy controls $(\mathrm{P}<0.05)$. We observed that the initial diameter of the OA was smaller in patients with NAION; thus, there would be decreased blood flow in the OA of these eyes, which may be related to hypoperfusion of the optic nerve. This finding provides some clues to the pathogenesis of NAION. However, there was no significant difference between uninvolved eyes of patients with NAION and healthy controls $(\mathrm{P}=0.31)$. There was no significant difference in the diameter of the initial ICA ( $\mathrm{P}=0.233)$ or the angle between the OA and ICA $(\mathrm{P}=0.424)$ across the three groups.

To calculate the mass flow of the initial OA, we first tried to develop a mesh of the OA and part of the ICA from two patients. The mass flow of the NAION-side OA accounted for ipsilateral ICA mass flow and was much lower than that on the uninvolved side. It is wellknown that approximately $15-25 \%$ of patients with NAION have bilateral optic neuropathy. We propose two 
explanations for our NAION model. The CTA data show real-time blood flow, which varies constantly. Increased blood flow in the uninvolved eye does not indicate that it will remain high. In contrast, autoregulation is the capacity to maintain a relatively constant level of blood flow in response to ocular perfusion pressure and varied metabolic demand. Many studies have demonstrated that efficient autoregulation of ocular blood flow in the ocular nerve head is mediated by increasing the capacitance of blood vessels. The changing magnitude of increased reactive vascular capacitance compensates for the decrease in vascular resistance of the ocular nerve head with increasing intraocular pressure (20). We speculate that autoregulation may play a role in ocular nerve head ischemia in patients with NAION, which may be compensated by an increase in blood flow, consequently leading to an increase in blood flow in the uninvolved eye. We verified the feasibility of this method, which calculates blood flow alterations.

In the NAION model, 3D velocity streamline results were red in color at the beginning of the OA on the uninvolved side, so the blood flow velocity was higher than that on the NAION side. The blood flow velocity at the initial OA in NAION eyes was lower and the diameters of the initial OA were relatively narrow. The lower blood flow velocity is also related to the regulation of intracranial vessels, and a narrower OA may be related to decreased vascular regulation or compensatory vasoconstriction. In our previous study using optical coherence tomography angiography, retinal vessel density and retinal thicknesses are also lower in NAION patients compared with healthy and uninvolved eyes (21). These may be associated with hypoperfusion of the underlying NAION eyes.

In this study, the accuracy of the $3 \mathrm{D}$ reconstruction models was limited by the slice thickness and hatch spacing of the CTA scan. Because the lumen of the OA is irregular and extremely thin, the branching pattern is complex, and they vary widely between individuals. Thus, we could not accurately calculate the blood flow to the optic nerve. We calculated the blood flow alterations in the three OA models. However, the present results need to be validated in a study with a larger sample size.

We generated 3D OA models and quantitatively estimated the $\mathrm{OA}$ diameter and the angle between the $\mathrm{OA}$ and ICA by comparing the eyes of healthy controls and patients with NAION. This is the first study to report that NAION is associated with a smaller initial OA diameter.
In addition, results from our preliminary analysis show blood flow alterations in the OA model generated using CTA data of a patient with NAION. This method could ultimately help identify the exact pathogenic mechanism of NAION.

\section{Acknowledgments}

Funding: This work was supported by the National Natural Science Foundation of China [No. 81870686]; the Research Foundation of Beijing Friendship Hospital, the Capital Medical University [yyqdkt 2019-29]; and Capital's Funds for Health Improvement and Research [No. 2018-1-2021].

\section{Footnote}

Reporting Checklist: The authors have completed the STROBE reporting checklist. Available at http://dx.doi. org/10.21037/atm-20-6883

Data Sharing Statement: Available at http://dx.doi. org/10.21037/atm-20-6883

Conflicts of Interest: All authors have completed the ICMJE uniform disclosure form (available at http://dx.doi. org/10.21037/atm-20-6883). The authors have no conflicts of interest to declare.

Ethical Statement: The authors are accountable for all aspects of the work in ensuring that questions related to the accuracy or integrity of any part of the work are appropriately investigated and resolved. The study was conducted in accordance with the Declaration of Helsinki (as revised in 2013). The study was approved by the ethics board of Beijing Friendship Hospital affiliated with Capital Medical University Ethics Committee (No. 2020-P2-00801 ) and informed consent was taken from all individual participants.

Open Access Statement: This is an Open Access article distributed in accordance with the Creative Commons Attribution-NonCommercial-NoDerivs 4.0 International License (CC BY-NC-ND 4.0), which permits the noncommercial replication and distribution of the article with the strict proviso that no changes or edits are made and the original work is properly cited (including links to both the formal publication through the relevant DOI and the license). See: https://creativecommons.org/licenses/by-nc-nd/4.0/. 


\section{References}

1. Newman NJ, Scherer R, Langenberg P, et al. The fellow eye in NAION: report from the ischemic optic neuropathy decompression trial follow-up study. Am J Ophthalmol 2002;134:317-28.

2. Hattenhauer MG, Leavitt JA, Hodge DO, et al. Incidence of nonarteritic anterior ischemic optic neuropathy. Am J Ophthalmol 1997;123:103-7.

3. Nazari H, Berry S, Sadaka A, et al. The challenge of managing NAION. Retina Specialist 2017;15:15-9.

4. Kerr NM, Chew SS, Danesh-Meyer HV. Non-arteritic anterior ischaemic optic neuropathy: a review and update. J Clin Neurosci 2009;16:994-1000.

5. Siesky B, Harris A, Kagemann L, et al. In vitro simulation of the first technique for non-invasive measurement of volumetric ophthalmic artery blood flow in humans. Ophthalmic Surg Lasers Imaging 2010;41:375-82.

6. Hedges TR. Ophthalmic artery blood flow in humans. $\mathrm{Br}$ J Ophthalmol 2002;86:1197.

7. Waugh JR, Sacharias N. Arteriographic complications in the DSA era. Radiology 1992;182:243-6.

8. Lian K, White JH, Bartlett ES, et al. NASCET percent stenosis semi-automated versus manual measurement on CTA. Can J Neurol Sci 2012;39:343-6.

9. Liu M, Sun A, Deng X. Hemodynamic performance within crossed stent grafts: computational and experimental study on the effect of cross position and angle. Biomed Eng Online 2018;17:85.

10. Kojima M, Irie K, Fukuda T, et al. The study of flow diversion effects on aneurysm using multiple enterprise stents and two flow diverters. Asian J Neurosurg 2012;7:159-65.

11. Sun A, Wang Z, Fan Z, et al. Influence of proximal drug eluting stent (DES) on distal bare metal stent (BMS) in multi-stent implantation strategies in coronary arteries. Med Eng Phys 2015;37:840-4.

Cite this article as: Wang JL, Wu DH, Li HY, Wang YL. Three-dimensional ophthalmic artery model reconstruction and assessment captured by computed tomographic angiography in non-arteritic anterior ischemic optic neuropathy patients. Ann Transl Med 2021;9(6):451. doi: 10.21037/atm-20-6883
12. Hayreh SS, Zimmerman B. Visual field abnormalities in nonarteritic anterior ischemic optic neuropathy: their pattern and prevalence at initial examination. Arch Ophthalmol 2005;123:1554-62.

13. Hunder GG, Bloch DA, Michel BA, et al. The American College of Rheumatology 1990 criteria for the classification of giant cell arteritis. Arthritis Rheum 1990;33:1122-8.

14. Patel DJ, Vaishnav RN. Basic hemodynamics and its role in disease processes. Baltimore: University Park Press, 1980.

15. Wang Z, Sun A, Fan Y, et al. Comparative study of Newtonian and non-Newtonian simulations of drug transport in a model drug-eluting stent. Biorheology 2012;49:249-59.

16. Harris FS, Rhoton AL. Anatomy of the cavernous sinus. A microsurgical study. J Neurosurg 1976;45:169-80.

17. Hayreh SS, Dass R. The ophthalmic artery: I. Origin and intra-cranial and intra-canalicular course. Br J Ophthalmol 1962;46:65-98.

18. Jiménez-Castellanos J, Carmona A, Castellanos L, et al. Microsurgical anatomy of the human ophthalmic artery: a mesoscopic study of its origin, course and collateral branches. Surg Radiol Anat 1995;17:139-43, 14-6.

19. Zhang T, Fan S, He W, et al. Ophthalmic artery visualization and morphometry by computed tomography angiography. Graefes Arch Clin Exp Ophthalmol 2015;253:627-31.

20. Riva CE, Hero M, Titze P, et al. Autoregulation of human optic nerve head blood flow in response to acute changes in ocular perfusion pressure. Graefes Arch Clin Exp Ophthalmol 1997;235:618-26.

21. Wang H, Meng Z, Li S, et al. Macular evaluation of the retinal and choroidal vasculature changes in anterior ischemic optic neuropathy-a case control study. BMC Ophthalmol 2018;18:341. 